\title{
El futuro de las editoriales universitarias en España
}

\author{
Antonio J. Baladrón-Pazos* \\ Beatriz Correyero-Ruiz**
}

Artículo recibido:

12 de noviembre de 2018

Artículo aceptado:

12 de marzo de 2019

Artículo de investigación

\section{Resumen}

En este trabajo los autores presentan una prospección sobre la evolución de las editoriales universitarias españolas a partir de un estudio Delphi realizado a cerca de una treintena de responsables de estos servicios editoriales, miembros de la Unión de Editoriales Universitarias Españolas (UNE). Los principales resultados obtenidos ponen de manifiesto la necesidad de un mayor reconocimiento del papel de las editoriales universitarias en el control y la promoción de la calidad de la edición académica. La adaptación al modelo digital, la gestión del open access, la flexibilización de los modelos de gestión para garantizar una mayor orientación al * Universidad Rey Juan Carlos de Madrid, España
Universidad Católica San Antonio de Murcia (UCAM), España
bcorreyero@ucam.edu
INVESTIGACIÓN BIBLIOTECOLÓGICA, vol. 33, núm. 81, octubre/diciembre, 2019, México,
ISSN: 2448-8321 pp. 179-200 
marketing y una mejor comercialización y distribución se plantean también como algunos de los retos más importantes.

Palabras clave: Editoriales Universitarias; Delphi; Investigación; España

\author{
The future of University Editorials in Spain \\ Antonio J. Baladrón-Pazos and Beatriz Correyero-Ruiz
}

\begin{abstract}
This research explores the evolution of Spanish University Editorials based on a Delphi study undertaken with an expert panel of about 30 board members of the Spanish University Presses Association (UNE). The main results obtained highlight the need for a greater recognition of the role played by university editorials in the control and promotion of the quality of scholarly publishing. The adaptation to the digital models, the management of open access, the flexibilization of business models to ensure greater marketing orientation and better marketing and distribution, are also considered as some of the most important challenges.
\end{abstract}

Keywords: University Press(es); Delphi; Research; Spain

\title{
INTRODUCCIÓN Y OBJETIVOS
}

E n este artículo se aborda el sector de las editoriales universitarias en Esna, un actor de indudable interés en el marco de la edición científica. Actualmente hay en todo el territorio nacional más de 3000 editoriales en activo que publican 90000 nuevos títulos cada año (MECD, 2018), datos que convierten a España en la quinta potencia europea en disponibilidad de títulos (658 086 títulos vivos) y la cuarta en número de nuevos lanzamientos anuales según la Federación de Editores Europeos (FED, 2016). Además, con un volumen de ventas aproximado de 2500 millones de euros al año, el mercado del libro español ocupa la novena posición en el ranking mundial, y si además relacionamos el volumen de ventas con el número de habitantes por 
país, España se sitúa en el sexto puesto, por detrás de Alemania, Noruega, Estados Unidos, Reino Unido y Australia (Ruediger Wischenbart Content and Consulting, 2017). En lo que respecta a la edición científica, existen 673 editoriales cuyas publicaciones son clasificadas en disciplinas como la Ciencia, la Tecnología, la Medicina, las Humanidades y las Ciencias Sociales (Giménez-Toledo, 2017b).

En España, la creación de la mayoría de los servicios de publicaciones, tal y como los conocemos en la actualidad, se produce en la década de 1980 en el contexto de la Ley de Reforma Universitaria de 1983; hasta ese momento, las universidades españolas publicaron apenas 2885 libros, mientras que en la década siguiente llegaron a más de 15000 títulos y en los seis primeros años del nuevo siglo eran ya 28000 (Pérez-Lasheras, 2007). Hoy en día las editoriales universitarias, en su mayoría asociadas a la Unión de Editoriales Universitarias Españolas (UNE), ${ }^{1}$ conforman el primer grupo de edición, ya que editan $34.03 \%$ de los libros académicos que se publican en España, suman en sus catálogos 58675 títulos vivos $^{2}$ y publican anualmente cerca de 4500 títu$\operatorname{los}^{3}$ (UNE, 2018). Atendiendo a las materias de publicación, $76.2 \%$ de la producción (3 424 títulos) son de Ciencias Sociales y Humanidades (UNE, 2018).

Las editoriales universitarias se diferencian de las editoriales comerciales en la filosofía que impulsa y motiva su publicación: apoyar a la docencia y divulgar la investigación, aunque cultivan muchos otros géneros (ensayos, actas de congresos o jornadas, traducciones, libros prácticos, etc). Giménez-Toledo añade que "no suelen tener afán de lucro y su actividad encuentra su razón de ser en un conjunto amplio de la sociedad a la que se dirigen mediante la publicación de obras de divulgación científica, y en los investigadores como creadores de contenido y como lectores de libros académicos” (2017a: 44). Las primeras editoriales universitarias modernas fueron Oxford y Cambridge, a mediados del siglo XVI. En cuanto a la historia de las editoriales universitarias españolas, Chartier (2007) fecha en 1494 el primer documento editado por una institución de enseñanza superior. Sin embargo, la proliferación de los servicios de publicaciones en las universidades

1 La UNE está integrada por 57 editorales y servicios de publicaciones de las universidades y 12 centros de investigación. De entre los proyectos desarrollados en los últimos años por esta asociación cabe destacar la creación de la plataforma de venta del libro universitario español (Unebook), la cual comercializa en la actualidad los 75000 libros que son fruto de la integración del fondo editorial de las universidades y centros de investigación, así como la edición de las revistas Unelibro y Unerevistas y la creación del Sello de Calidad para Colecciones Académicas CEA-APQ.

2 De ellos, $61.1 \%$ (35 862) son monografías y $38.9 \%$ se distribuye entre manuales (9 898), revistas científicas (6 506), tesis y microficas (5 705) y apuntes (704).

3 Según la última edición del informe Las editoriales universitarias en cifras 2017 (UNE, 2018), en el último año contabilizado (2017) los sellos universitarios publicaron 4497 títulos, un $4 \%$ menos que en 2015. De ellos, 3732 fueron novedades ( $83 \%$ ) y 765 reediciones (17 \%). 
se inició en el siglo XIX. Empezó en Estados Unidos con editoriales como John Hopkins, Princeton, Harvard o Standford y, más tarde, se extendió a Europa y Latinoamérica.

La eclosión de la edición universitaria en España producida durante las dos últimas décadas se ha desarollado paralela a una profunda transformación del sector: se ha avanzado en la profesionalización de sus estructuras, se ha incorporado el diseño a sus productos o se ha aumentado notablemente la edición digital, casi $600 \%$ en los últimos años, llegando a representar en $201733 \%$ de los títulos editados por las universidades (UNE, 2018). Junto a ello, el crecimiento de la competencia y de la oferta editorial se ha producido en un contexto de estancamiento de la demanda, motivado principalmente por el hecho de que los estudiantes cada vez usan más los apuntes o fuentes gratuitas de internet y porque los docentes no promueven la compra de libros (Gómez-Hernández, 2007). Esta situación ha incidido principalmente en dos factores: la reducción de las tiradas y el recorte del presupuesto. Según la UNE (2018), en el último año contabilizado (2017), se editaron 1110541 ejemplares, lo cual supuso 25.4 \% menos que en 2015. En 2017 el presupuesto global del funcionamiento fue de 12142462 euros, registrando una disminución de 16.8 \% respecto a 2015 . De la asignación presupuestaria, $68.5 \%$ proviene de la propia universidad, $25.8 \%$ de recursos propios y $5.7 \%$ de recursos ajenos; esta última partida es la que ha experimentado un mayor descenso en los últimos 10 años.

La edición universitaria se encuentra, por tanto, en un momento de transformación que hace necesarios estudios que profundicen no sólo en su situación actual sino también en sus perspectivas de futuro. En este marco se sitúa la investigación que aquí presentamos y que tiene como telón de fondo el aumento en los últimos años del interés académico por el estudio de las editoriales universitarias a nivel nacional e internacional. En el caso de América Latina destacan los trabajos de Canossa-Mendes y Córdoba-Restrepo (2011), Córdoba-Restrepo (2014), Sagastizábal et al. (2016), Costa y Sagastizábal (2016), y Giménez-Toledo, Tejada-Artigas y Mañana-Rodríguez (2018). Por su parte, se puede hacer una radiografía de las editoriales universitarias norteamericanas gracias a los trabajos de, por ejemplo, Whitey et al. (2011), Clare (2014), Morotti (2015), Watkinson (2016), y Aldana (2018). En cuanto a las investigaciones europeas, son numerosos los trabajos sobre las editoriales universitarias británicas (Jubb, 2017; Deegan 2017; Lockett y Speicher, 2016; Cond, 2016; Keene et al., 2016; Rayner, 2016), alemanas (Bargheer y Pabst, 2016) o francesas (Henny, 2015). Los estudios también ofrecen información sobre África (Luescher y Van Schalkwyk, 2018) e India (Das, 2015). En el caso de España, encontramos investigaciones de carácter general sobre las editoriales universitarias (Abadal-Falgueras y Ollé-Castellà, 
2012; Borcha, 2012; Polo-Pujadas, 2007; Giménez-Toledo, 2016) pero proliferan también aquellos estudios bien centrados en aspectos concretos que representan las principales preocupaciones del sector como son la edición digital (Cordón-García, 2014; Cordón-García et al., 2014), el acceso abierto (Abadal-Falgueras, Ollé-Castellà y Redondo, 2018), la calidad y el prestigio editorial (Giménez-Toledo, 2018; Giménez-Toledo 2017a; Giménez-Toledo, Mañana-Rodríguez y Sivertsen, 2017; Giménez-Toledo, Mañana-Rodríguez y Tejada-Artigas, 2015; Delgado López-Cózar, 2014; Álvarez, 2012; Fernández-Beltrán, 2011; Cordón-García y Gómez-Díaz, 2010) o la evaluación comparada (Mañana-Rodríguez y Giménez-Toledo, 2017; Mañana-Rodríguez y Giménez-Toledo, 2016; Giménez-Toledo et al., 2016).

El objetivo de la investigación llevada a cabo fue realizar una prospectiva, fundamentada científicamente, de las editoriales universitarias en España para los próximos 10 años. En el marco de este objetivo general, se establecieron los siguientes objetivos específicos: conocer el papel que dichas editoriales jugarán en el contexto de la investigación y la educación superior; identificar las debilidades y fortalezas que les dificultarán o facilitarán, respectivamente, su competitividad, y prever las medidas que adoptarán para consolidarse como referencia en el mercado de la edición científico-técnica. Estos objetivos guían el planteamiento metodológico de nuestra investigación, respaldado por el trazado argumentativo sobre el importante papel que las editoriales universitarias cumplen en el marco del sector editorial español y sobre el que se ha incidido en esta introducción como contexto de los resultados obtenidos y que se expondrán en las próximas líneas.

\section{Metodología}

Puesto que los objetivos anteriormente expuestos se refieren a la evolución futura de las editoriales universitarias, se optó por el método Delphi, una técnica de previsión subjetiva acorde a los objetivos de nuestra investigación, con la que no se perseguía describir la situación de las editoriales universitarias sino principalmente detectar tendencias de futuro. A diferencia de otras técnicas de previsión individuales ( $v$.gr., encuestas), el método Delphi, como consecuencia del feedback sobre el que se basa, permite que los resultados obtenidos sean producto de la interacción de un grupo de personas, siendo el resultado grupal superior a la suma de las aportaciones individuales de los participantes. Asimismo, frente a otras técnicas grupales como los focus group, en este caso el anonimato entre los participantes evita influencias negativas de los miembros dominantes, que podrían orientar los resultados obtenidos. 
El Delphi es un método consistente en un proceso iterativo y sistemático en el que un grupo de expertos en el tema analizado es interrogado en diferentes rondas con el fin de obtener un consenso de opinión o, al menos, una opinión grupal fidedigna. Para la conformación del panel de expertos se optó por los responsables de editoriales universitarias en España, objeto de estudio de nuestra investigación, si bien están en marcha otras fases en las que se colabora con otro perfil de participantes. En esta primera fase se optó por los mismos dado que se buscaba trabajar con especialistas en el tema y que además estuviesen implicados activamente en él, además de que cumpliesen otros criterios que garantizaran el logro de los objetivos planteados, tales como su nivel de conocimientos, su capacidad predictiva y su grado de motivación. En total iniciaron el proceso los siguientes 27 expertos: Alfonso Martín Jiménez, Director, Ediciones Universidad de Valladolid; Alfonso Pinilla García, Director del Servicio de Publicaciones, Universidad de Extremadura; Ana Rodríguez de Agüero Delgado, Directora, CEU Ediciones; Antonio López Fonseca, Director, Ediciones Complutenses; Benjamín González Díaz, Director, Servicio de Publicaciones, Universidad de La Laguna; Consuelo Martínez Moraga, Directora del Departamento de Publicaciones, Editorial Universidad Francisco de Vitoria; Edurne Aranguren Alonso, Técnica de Publicaciones y Secretaria del Consejo Editorial, Unidad de Publicaciones, Universidad de Navarra; Felipe del Pozo Redondo, Director del Centro de Recursos para el Aprendizaje y la Investigación y Director de la Biblioteca de la UNIA, Servicio de Publicaciones, Universidad Internacional de Andalucía; Fernando Díez Ruiz, Director, Oficina de Transferencia de Conocimiento y Publicaciones, Deusto Publicaciones; Francisca Llabres Segura, Directora Técnica de Publicaciones, Ediciones Universidad de las Islas Baleares; Francisco Antonio Gil Pujante, Director Técnico del Servicio de Publicaciones, Ediciones Universidad de Murcia; Francisco Fernández Beltrán, Director de Comunicación y Publicaciones, Universidad Jaume I; Gerardo Marraud González, Director Biblioteca Universitaria, Servicio de Publicaciones, Universidad de Vigo; Javier Balibrea, Director Gerente, Ediciones Universidad de Navarra; Joan Carles Marset, Director del Servicio de Publicaciones, Universidad Autónoma de Barcelona; Jordi Prats, Director, Oficina de Publicaciones Académicas Digitales, Universidad Politécnica de Cataluña; José Beltrán Fortes, Director, Editorial Universidad de Sevilla; José Luis Canet, Director del Servicio de Publicaciones, Publicaciones de la Universidad de Valencia; José Manuel Trabado Cabado, Director del Área de Publicaciones, Publicaciones Universidad de León; José Marchena Domínguez, Director General de Extensión Cultural y Servicio de Publicaciones, Editorial Universidad de Cádiz; Juan Carlos Valderrama, Director del Servicio de Publicaciones, Universidad Católica de 
Valencia; Juan Luis Blanco Valdés, Director, Servicio de Publicaciones e Intercambio Científico de la Universidad de Santiago de Compostela; Juan Pedro Monferrer Sala, Director, UCOPress Universidad de Córdoba; María Remedios Pérez García, Jefa de Unidad Administrativa, Editorial Universidad Politécnica de Valencia; Marina Díaz Peralta, Directora de Comunicación y del Servicio de Publicaciones y Difusión Científica, Servicio de Publicaciones y Difusión Científica, Universidad de las Palmas de Gran Canaria; Olivia Manzanaro García, Responsable de Proyectos Digitales, Publicaciones Universidad de Alicante; Teresa Agirreazaldegi Berriozabal, Directora del Servicio de Publicaciones, Universidad del País Vasco.

En el momento de hacer esta investigación, la UNE contaba con 57 servicios editoriales de universidades asociadas, con lo que el panel de expertos participantes en nuestra investigación incluye $47.3 \%$; aunque el método Delphi no exige representatividad en la muestra de participantes es importante destacar esa alta participación debida a la excelente acogida que tuvo nuestra investigación, y que además se complementa con la significatividad del panel de expertos. Por otra parte, el índice de abandono en los trabajos publicados suele oscilar entre 20 y 30 \% (Landeta, 2002: 122), mientras que en este caso apenas supuso $3.7 \%$, por lo que, de acuerdo a la ortodoxia del método Delphi, dicho índice no implicó distorsión en los resultados finales obtenidos.

Se llevaron a cabo dos rondas de consultas mediante cuestionarios enviados por correo electrónico durante los meses de mayo y junio de 2018. El primer cuestionario estaba constituido por cinco preguntas abiertas, de las cuales las cuatro primeras fueron las que se incorporaron al proceso iterativo en coherencia con los objetivos planteados en la investigación. A partir de las contestaciones obtenidas se extrajeron, mediante un proceso de integración de las opiniones individuales de los expertos en una estimación grupal, los ítems de respuesta del cuestionario de la segunda ronda, conformado por cuatro preguntas cerradas y 112 ítems de respuesta ordenados alfabéticamente para evitar condicionamientos en la valoración de los mismos. Se solicitaron valuaciones por parte de los expertos en una escala 0-5 ya que, frente a la jerarquización, la valuación facilitaba la respuesta de los expertos dado el elevado número de ítems en cada pregunta. Con esta segunda ronda se puso fin al proceso iterativo, de acuerdo al criterio de parada establecido previamente en el diseño metodológico de la investigación con el fin de evitar la desmotivación de los expertos participantes; de hecho, diferentes razones justifican que rara vez se alcance una tercera ronda en los estudios Delphi a pesar de que no se consiga totalmente el consenso o la estabilidad en las respuestas (Landeta, 2002: 101-102). En cualquier caso, se garantizaban los tres niveles discursivos 
del método Delphi (Gaitán-Moya y Piñuel-Raigada, 1998: 138-139): el distribucional, referido a la construcción del discurso de cada experto mediante la respuesta a los cuestionarios; el relacional, al acoplamiento del discurso particular de los expertos con respecto al de los copartícipes en el proceso, y el integrativo, al papel de los investigadores responsables del estudio en el control del feedback y la síntesis de los distintos discursos particulares. A continuación, se sometieron las respuestas del segundo cuestionario a tratamiento estadístico, calculando la mediana como medida de tendencia central de los valores de cada ítem en cada una de las preguntas ya que la media daría demasiado peso a las respuestas extremas, pudiendo desvirtuarse la opinión grupal.

\section{RESULTADOS Y DisCUSIÓN}

Una vez explicado el planteamiento metodológico de la investigación realizada, en este apartado se exponen los principales resultados obtenidos. En las tablas se ordenan los distintos ítems de respuesta de mayor a menor consenso para una mayor claridad en la exposición de los datos, es decir: de mediana superior a inferior, y en caso de igual mediana, de menor a mayor desviación típica.

\section{Misión de las editoriales universitarias}

En primer lugar, se interrogó a los participantes en el Delphi sobre la misión que las editoriales universitarias españolas tendrán en el contexto de la educación y la investigación superior. Estas editoriales desempeñarán un papel fundamental en el control y la promoción de la calidad en la edición científico-técnica en este país, lo cual permitirá -según los expertos- un mayor reconocimiento de las editoriales universitarias bien mediante sellos de calidad o bien mediante su indexación en otros referentes de calidad. Asimismo, las editoriales universitarias tendrán como misión también fundamental la transferencia a la sociedad de la investigación realizada en las universidades, el impulso a nuevas formas de acceso al conocimiento propiciadas por el nuevo modelo digital y la elaboración de materiales didácticos, tanto tradicionales como interactivos, adaptados a las demandas de la docencia universitaria. Precisamente sobre su papel en el nuevo modelo digital, el impulso a la difusión en abierto del conocimiento científico es otra de las misiones que 
señalan los expertos participantes, si bien en este grado el nivel de disenso es algo superior, por lo que estos datos han de ser contrastados con las opiniones vertidas en el resto de preguntas del cuestionario y que se expondrán en las próximas líneas, por ejemplo, cuando se abordan las debilidades de estas editoriales para ser competitivas en esos modelos digitales o cuando los expertos opinan sobre la conveniencia de promover el acceso abierto de los contenidos. Por último, los expertos conceden una menor relevancia a otras misiones que corresponderán a estas editoriales, principalmente las que tienen que ver con potenciar la visibilidad de las universidades en la sociedad, la divulgación y formación de los ciudadanos o incluso el debate social, temas culturales y científicos, así como otras más internas como su papel al servicio de los autores o la necesaria compatibilización entre la misión universitaria y la rentabilidad en los mercados de sus productos editoriales. En la Tabla 1 se recoge el detalle de los diferentes ítems de respuesta.

\begin{tabular}{|c|c|c|c|c|}
\hline Puesto & Ítem & Mediana & Media & Desv. \\
\hline 1 & $\begin{array}{l}\text { Maximizar la calidad de las publicaciones, verificándola por } \\
\text { ejemplo mediante sellos de calidad }\end{array}$ & 5 & 4.65 & 0.55 \\
\hline 2 & $\begin{array}{l}\text { Controlar la calidad y la transparencia en los procesos de } \\
\text { selección editorial }\end{array}$ & 5 & 4.57 & 0.63 \\
\hline 3 & $\begin{array}{l}\text { Participar activamente en el cambio hacia el modelo digital, } \\
\text { impulsando nuevas formas de acceso al conocimiento }\end{array}$ & 5 & 4.53 & 0.69 \\
\hline 3 & $\begin{array}{l}\text { Transferir a la sociedad la investigación que se realiza en las } \\
\text { universidades }\end{array}$ & 5 & 4.5 & 0.69 \\
\hline 4 & $\begin{array}{l}\text { Ser referencia académica para la publicación de investigación } \\
\text { de calidad }\end{array}$ & 5 & 4.42 & 0.74 \\
\hline 5 & $\begin{array}{l}\text { Proporcionar materiales didácticos para la docencia universi- } \\
\text { taria, tanto tradicionales como interactivos } \\
\text { y multimedia }\end{array}$ & 5 & 4.26 & 0.86 \\
\hline 6 & $\begin{array}{l}\text { Potenciar la indexación y el reconocimiento de las revistas } \\
\text { científicas }\end{array}$ & 5 & 4.38 & 0.96 \\
\hline 7 & $\begin{array}{l}\text { Impulsar la difusión en acceso abierto del conocimiento } \\
\text { científico }\end{array}$ & 5 & 4.32 & 1.26 \\
\hline 8 & $\begin{array}{l}\text { Potenciar el prestigio y la visibilidad de las universidades en } \\
\text { la sociedad }\end{array}$ & 4 & 4.46 & 0.5 \\
\hline 9 & Promover la divulgación y la formación de los ciudadanos & 4 & 3.92 & 0.83 \\
\hline 10 & $\begin{array}{l}\text { Incentivar el debate social acerca de temas culturales } \\
\text { y científicos }\end{array}$ & 4 & 4.07 & 0.87 \\
\hline 11 & $\begin{array}{l}\text { Asesorar a los autores y/o lectores en materia de derechos } \\
\text { de autor }\end{array}$ & 4 & 4 & 0.88 \\
\hline 11 & $\begin{array}{l}\text { Implantar en la edición universitaria modelos de gestión que } \\
\text { compatibilicen la misión universitaria con la rentabilidad }\end{array}$ & 4 & 3.61 & 0.88 \\
\hline
\end{tabular}




\begin{tabular}{|c|l|c|c|c|}
\hline 12 & $\begin{array}{l}\text { Incrementar la colaboración entre universidades y otras } \\
\text { instituciones }\end{array}$ & 4 & 3.92 & 1.06 \\
\hline 13 & Fomentar la investigación científica & 4 & 3.62 & 1.11 \\
\hline 14 & $\begin{array}{l}\text { Fomentar la internacionalización de la institución a la que } \\
\text { pertenecen }\end{array}$ & 4 & 3.88 & 1.12 \\
\hline 15 & $\begin{array}{l}\text { Diversificar los productos editoriales impulsados desde las } \\
\text { universidades }\end{array}$ & 4 & 3.61 & 1.18 \\
\hline 16 & $\begin{array}{l}\text { Contribuir a la promoción de los jóvenes docentes } \\
\text { e investigadores }\end{array}$ & $\begin{array}{l}\text { Intensificar la colaboración entre las editoriales universitarias } \\
\text { y las privadas }\end{array}$ & 3.76 & 1.22 \\
\hline 17 & 3.36 & 1.13 \\
\hline
\end{tabular}

Tabla 1. Misión de las editoriales universitarias

Fuente: elaboración propia

\section{Debilidades}

Esta misión de las editoriales universitarias podría verse influida por algunas de las principales debilidades que dificultarán en los próximos 10 años la competitividad de las mismas tanto en el mercado nacional como en el internacional. Como se puede apreciar en la Tabla 2, en este caso los niveles de disenso entre los participantes en el Delphi son mucho más elevados, lo que pudiera deberse a la diversidad de modelos de editoriales universitarias existentes y a la tendencia de los expertos a focalizar las respuestas en las debilidades de su propio modelo y no tanto en las del sector de las editoriales universitarias españolas en general. En cualquier caso, el principal problema al que seguirán enfrentándose es exógeno; la falta de reconocimiento de las editoriales por parte de los organismos evaluadores de la trayectoria académica e investigadora de los profesores, ya que en los últimos años la tendencia ha sido la de priorizar las revistas sobre los libros como canales de difusión de la investigación científica. Junto a él, los expertos señalan otras dificultades endógenas, principalmente las siguientes: escasos recursos humanos y plantillas inadecuadas, problemas de financiación, recursos materiales o apoyo institucional, y modelos deficientes de comercialización y distribución. Precisamente, tanto respecto a las debilidades exógenas como a las endógenas, los expertos también apuntan otras en las que los grados de consenso son variables, pero sin duda conectan con las principales que hemos referido anteriormente. Así, en cuanto a lo exógeno, diferentes ítems de respuesta se refieren al cuestionamiento de los modelos de evaluación de la calidad de la producción científica y el bajo posicionamiento de las editoriales universitarias en esos indicadores de calidad, así como a la menor valoración 
en el mundo académico de estas editoriales frente a las editoriales privadas, integradas en multinacionales con un perfil más comercial y altamente competitivas. Respecto a lo endógeno, las debilidades referidas se completan con otras como la burocratización y falta de flexibilidad en la gestión de estas editoriales universitarias, que enraíza en una excesiva dependencia de los órganos rectores de las universidades lo cual seguirá dificultando en los próximos 10 años su competitividad. En la Tabla 2 se recogen otros ítems de respuesta con menor valoración y destacados niveles de disenso, que por razones de espacio y para no ser reiterativos no podemos comentar en detalle. Algunos de ellos se refieren a debilidades que aparentemente pudiera pensarse que ponen en peligro algunas de las misiones que según los expertos desempeñarán estas editoriales universitarias en los próximos años, sobre todo las que tienen que ver con las débiles infraestructuras y políticas para ser competitivas en el nuevo modelo digital o las dificultades que existirán para combinar el acceso abierto con la necesaria rentabilidad de estas editoriales.

\begin{tabular}{|c|c|c|c|c|}
\hline Puesto & Ítem & Mediana & Media & Desv. \\
\hline 1 & $\begin{array}{l}\text { Falta de reconocimiento de los organismos evaluadores del } \\
\text { profesorado, que priorizan las revistas como plataforma de } \\
\text { difusión científica }\end{array}$ & 5 & 4.15 & 1.29 \\
\hline 2 & Escasos recursos humanos y/o plantillas inadecuadas & 4.5 & 4 & 1.27 \\
\hline 3 & $\begin{array}{l}\text { Falta de recursos materiales, apoyo institucional y problemas } \\
\text { de financiación }\end{array}$ & 4 & 3.88 & 1.05 \\
\hline 4 & $\begin{array}{l}\text { Distribución y comercialización deficiente y/o carente de } \\
\text { estrategia }\end{array}$ & 4 & 3.88 & 1.08 \\
\hline 5 & $\begin{array}{l}\text { Sometimiento a indicadores de calidad que no siempre } \\
\text { valoran la evaluación rigurosa de originales y/o conducen a } \\
\text { jerarquización de las editoriales }\end{array}$ & 4 & 3.76 & 1.09 \\
\hline 6 & Competencia feroz de los grupos editoriales multinacionales & 4 & 3.92 & 1.13 \\
\hline 7 & $\begin{array}{l}\text { Burocratización y estructuras de gestión y toma de decisiones } \\
\text { poco flexibles }\end{array}$ & 4 & 3.69 & 1.14 \\
\hline 8 & $\begin{array}{l}\text { Reducidas colecciones con sellos de calidad, que son las } \\
\text { valoradas por los organismos evaluadores, y baja posición en } \\
\text { los rankings de calidad }\end{array}$ & 4 & 3.69 & 1.23 \\
\hline 9 & $\begin{array}{l}\text { Excesiva dependencia de los órganos rectores de las univer- } \\
\text { sidades, que dificultan la competitividad y el diseño de planes } \\
\text { estratégicos a largo plazo }\end{array}$ & 4 & 3.30 & 1.26 \\
\hline 10 & $\begin{array}{l}\text { Alta identificación con formatos de publicación tradicionales y } \\
\text { necesidad de crear nuevos modelos de acceso al conoci- } \\
\text { miento y servicios de valor añadido }\end{array}$ & 4 & 3.46 & 1.28 \\
\hline 11 & $\begin{array}{l}\text { Menor valoración en el mundo académico frente a las } \\
\text { editoriales privadas }\end{array}$ & 4 & 3.73 & 1.37 \\
\hline
\end{tabular}




\begin{tabular}{|c|c|c|c|c|}
\hline 12 & Perfil poco comercial frente a las editoriales privadas & 4 & 3.26 & 1.48 \\
\hline 13 & $\begin{array}{l}\text { Nivel de innovación insuficiente para las exigencias del } \\
\text { mercado }\end{array}$ & 3.5 & 3.19 & 1.21 \\
\hline 14 & $\begin{array}{l}\text { Necesidad de mejor adaptación a los nuevos hábitos de } \\
\text { consumo y lectura }\end{array}$ & 3.5 & 3.34 & 1.24 \\
\hline 15 & $\begin{array}{l}\text { Falta de apertura al mercado internacional, influida por la } \\
\text { edición en castellano }\end{array}$ & 3.5 & 3.23 & 1.28 \\
\hline 16 & Insuficiente hábito lector de la sociedad en general & 3.5 & 3.30 & 1.32 \\
\hline 17 & $\begin{array}{l}\text { Infraestructuras y políticas débiles para competir en el ámbito } \\
\text { de la edición y comercialización digital }\end{array}$ & 3.5 & 3.07 & 1.36 \\
\hline 18 & $\begin{array}{l}\text { Dificultades para conjugar las políticas de acceso abierto con } \\
\text { la rentabilidad }\end{array}$ & 3.5 & 3.26 & 1.37 \\
\hline 19 & Descuido de la promoción y la gestión de la comunicación & 3 & 3.38 & 1.21 \\
\hline 20 & $\begin{array}{l}\text { Insuficiente colaboración con otras editoriales universitarias, } \\
\text { pese a la importante función que cumple la UNE }\end{array}$ & 3 & 2.76 & 1.22 \\
\hline 21 & $\begin{array}{l}\text { Poca colaboración con otras editoriales e instituciones } \\
\text { internacionales }\end{array}$ & 3 & 3.15 & 1.29 \\
\hline 22 & $\begin{array}{l}\text { Problemas para compatibilizar la viabilidad económica con } \\
\text { publicaciones científicas muy especializadas, de alta calidad } \\
\text { y públicos restringidos }\end{array}$ & 3 & 3.42 & 1.31 \\
\hline 23 & $\begin{array}{l}\text { Diversidad de modelos de editoriales universitarias y falta de } \\
\text { criterios comunes }\end{array}$ & 3 & 2.84 & 1.32 \\
\hline 24 & $\begin{array}{l}\text { Problemas de demanda en el modelo Bolonia, que ha desin- } \\
\text { centivado la utilización de manuales en las clases }\end{array}$ & 3 & 2.96 & 1.34 \\
\hline 25 & $\begin{array}{l}\text { Imagen de estas editoriales como apéndices de la edición } \\
\text { institucional }\end{array}$ & 3 & 3.11 & 1.37 \\
\hline 26 & $\begin{array}{l}\text { Deficiente número de publicaciones del área de las cc. } \\
\text { experimentales y salud }\end{array}$ & 3 & 3.32 & 1.38 \\
\hline 26 & Incapacidad para luchar contra la piratería & 3 & 2.69 & 1.38 \\
\hline 27 & Políticas deficientes para la gestión de metadatos & 3 & 2.88 & 1.42 \\
\hline 28 & $\begin{array}{l}\text { Endogamia o escasa capacidad para atraer a autores } \\
\text { externos }\end{array}$ & 3 & 3.19 & 1.44 \\
\hline 29 & $\begin{array}{l}\text { Elevado número de títulos y bajas tiradas medias, lo que } \\
\text { dificulta la rentabilidad }\end{array}$ & 3 & 2.80 & 1.49 \\
\hline 29 & $\begin{array}{l}\text { Excesiva dependencia de los sistemas de evaluación de la } \\
\text { calidad }\end{array}$ & 3 & 2.73 & 1.53 \\
\hline 31 & $\begin{array}{l}\text { Debilidad del modelo por las medidas gubernamentales } \\
\text { que obligarán a depositar en repositorios institucionales la } \\
\text { investigación hecha con fondos públicos }\end{array}$ & 3 & 3.15 & 1.61 \\
\hline
\end{tabular}

Tabla 2. Debilidades de las editoriales universitarias

Fuente: elaboración propia 


\section{Principales fortalezas}

Respecto a las fortalezas que en los próximos 10 años contribuirán a la competitividad de las editoriales universitarias españolas en el mercado nacional e internacional, los expertos subrayan principalmente su trayectoria y experiencia en la edición científica y la evaluación rigurosa que llevan a cabo para la selección de los originales. El acuerdo es algo menor en cuanto a la tercera fortaleza, la potenciación del acceso abierto a los contenidos; en esta cuestión, del estudio realizado se deducen plantemientos diversos por parte de los expertos participantes ya que las opiniones agregadas reflejan, como acabamos de comentar, la dificultad de combinar el acceso abierto con la rentabilidad pero también la potenciación de ese acceso abierto como una de sus principales fortalezas de cara a ser competitivas y cumplir así con una de las que consideran sus principales misiones. Los índices de disenso registrados en los ítems de respuesta referidos al acceso abierto en las diferentes preguntas planteadas en los cuestionarios del proceso iterativo Delphi pueden servir para explicar esas aparentes divergencias, que en todo caso apuntan a la potenciación del mismo en el futuro de las editoriales universitarias españolas, tal y como más adelante se reiterará. En otro orden de cosas, son también fortalezas importantes señaladas por las editoriales universitarias para los próximos años las siguientes: sus alianzas con bibliotecas y otros ámbitos universitarios; su especialización, diversidad y actualización de los fondos editoriales; su cultura de calidad y buenas prácticas editoriales, que contribuirán a un progresivo cumplimiento de estándares de calidad y obtención de certificaciones y sellos, así como presencia en bases de datos internacionales; su proximidad a la comunidad científica como principales clientes, su competencia cada vez mayor en la edición de revistas científicas, sus precios asequibles o su importante presencia en el mercado latinoamericano. Otro de los puntos fuertes subrayados por los expertos se contrapone a las opiniones vertidas en anteriores respuestas. Por ejemplo, la debilidad referida a los recursos humanos de estas editoriales se compensa aquí con la referencia a la profesionalidad creciente de los equipos editores o a los consejos editoriales de alto nivel, cuestión que también se considera esencial cuando los expertos son interrogados por las medidas que adoptarán las editoriales universitarias en los próximos años. Cierta contradicción se observa cuando la falta de recursos económicos y apoyo institucional se consideraba una debilidad de estas editoriales, mientras que en las respuestas a esta pregunta el respaldo económico e institucional de las universidades también se apunta como una fortaleza destacada. En este sentido serían necesarios estudios complementarios que abordasen esta posible contradición. En cualquier caso, en la Tabla 3 se puede obsevar que, grosso modo, 
en la opinión agregada de los expertos respecto a las fortalezas se han logrado unos mayores grados de consenso que en el caso de la opinión respecto a las debilidades.

\begin{tabular}{|c|c|c|c|c|}
\hline Puesto & Ítem & Mediana & Media & Desv. \\
\hline 1 & Experiencia en la edición científica & 4.5 & 4.34 & 0.73 \\
\hline 2 & Evaluación externa y rigurosa en la selección de originales & 4.5 & 4.26 & 0.81 \\
\hline 3 & Potenciación del acceso abierto a los contenidos & 4.5 & 4.15 & 1.17 \\
\hline 4 & $\begin{array}{l}\text { Posibilidad de alianzas con las bibliotecas y otras unidades } \\
\text { universitarias }\end{array}$ & 4 & 3.96 & 0.71 \\
\hline 5 & $\begin{array}{l}\text { Riqueza, diversidad y actualización de títulos en diversas } \\
\text { áreas de conocimiento }\end{array}$ & 4 & 4.23 & 0.75 \\
\hline 6 & $\begin{array}{l}\text { Progresivo reconocimiento de estándares, certificaciones } \\
\text { y sellos de calidad }\end{array}$ & 4 & 4.11 & 0.85 \\
\hline 6 & $\begin{array}{l}\text { Proximidad a la comunidad científica como principales } \\
\text { clientes }\end{array}$ & 4 & 4.11 & 0.85 \\
\hline 7 & Competencia en la gestión de revistas científicas & 4 & 3.73 & 0.90 \\
\hline 8 & $\begin{array}{l}\text { Cultura de calidad, buenas prácticas de edición y mejora } \\
\text { continua }\end{array}$ & 4 & 4.15 & 0.91 \\
\hline 9 & Presencia en bases de datos internacionales & 4 & 4.19 & 0.92 \\
\hline 10 & Especialización de sus fondos editoriales & 4 & 4.07 & 0.96 \\
\hline 10 & Precios asequibles de sus publicaciones & 4 & 3.92 & 0.96 \\
\hline 11 & $\begin{array}{l}\text { Importante influencia en el mercado internacional sobre } \\
\text { todo latinoamericano, por la edición en castellano }\end{array}$ & 4 & 3.96 & 0.98 \\
\hline 12 & Profesionalidad creciente de los equipos editores & 4 & 4 & 1.00 \\
\hline 13 & $\begin{array}{l}\text { Acceso privilegiado a los autores, que son productores de } \\
\text { contenido de alto nivel }\end{array}$ & 4 & 4.07 & 1.03 \\
\hline 13 & $\begin{array}{l}\text { Papel estratégico de la edición universitaria para el } \\
\text { posicionamiento de la investigación y el prestigio de las } \\
\text { universidades }\end{array}$ & 4 & 3.92 & 1.03 \\
\hline 14 & $\begin{array}{l}\text { Competitividad en la creación de materiales docentes } \\
\text { tradicionales y MOOC }\end{array}$ & 4 & 3.36 & 1.05 \\
\hline 15 & Promoción y respeto por los derechos de autor & 4 & 3.96 & 1.06 \\
\hline 16 & $\begin{array}{l}\text { Incremento en las actividades de comunicación y relacio- } \\
\text { nes públicas (ferias, etc.) }\end{array}$ & 4 & 3.65 & 1.07 \\
\hline 17 & $\begin{array}{l}\text { Imagen de prestigio de la institución universitaria que se } \\
\text { traslada a sus editoriales }\end{array}$ & 4 & 3.88 & 1.09 \\
\hline 17 & Respaldo económico e institucional de las universidades & 4 & 4.03 & 1.09 \\
\hline
\end{tabular}




\begin{tabular}{|c|c|c|c|c|}
\hline 18 & $\begin{array}{l}\text { Intercambio electrónico que permite la disponibilidad en } \\
\text { todas las bibliotecas universitarias con inmediatez y bajo } \\
\text { coste }\end{array}$ & 4 & 3.88 & 1.15 \\
\hline 19 & Consejos editoriales de alto nivel & 4 & 3.92 & 1.21 \\
\hline 20 & $\begin{array}{l}\text { Asociación institucional de las editoriales universitarias, } \\
\text { marca UNE }\end{array}$ & 4 & 3.73 & 1.26 \\
\hline 21 & $\begin{array}{l}\text { Tendencia al alza en la edición digital, que permite mayor } \\
\text { adaptación a la demanda y menores costes }\end{array}$ & 4 & 3.88 & 1.31 \\
\hline 22 & $\begin{array}{l}\text { Creación de plataformas virtuales conjuntas de comerciali- } \\
\text { zación como UNEbook }\end{array}$ & 4 & 3.69 & 1.32 \\
\hline 23 & $\begin{array}{l}\text { Concepción no mercantilista de la actividad editorial vs. } \\
\text { editoriales privadas }\end{array}$ & 4 & 3.69 & 1.46 \\
\hline 24 & Calidad en el diseño y ediciones atractivas & 3.5 & 3.46 & 1.08 \\
\hline 25 & $\begin{array}{l}\text { Colaboraciones entre las editoriales universitarias y las } \\
\text { editoriales privadas }\end{array}$ & 3 & 2.76 & 1.12 \\
\hline 26 & $\begin{array}{l}\text { Capacidad de adaptación, innovación y orientación al } \\
\text { cambio }\end{array}$ & 3 & 3.34 & 1.14 \\
\hline 27 & $\begin{array}{l}\text { Alianzas con los organismos evaluadores del profesorado, } \\
\text { que ponen en valor la edición universitaria }\end{array}$ & 3 & 3.38 & 1.15 \\
\hline 28 & Cobro de derechos reprográficos & 2 & 2.34 & 1.11 \\
\hline
\end{tabular}

Tabla 3. Fortalezas de las editoriales universitarias

Fuente: elaboración propia

\section{Medidas que se adoptarán en el futuro}

Para terminar, se preguntó a los expertos participantes acerca de las medidas que en los próximos 10 años adoptarán las editoriales universitarias en España para consolidarse como editoriales de referencia. Algunas de las respuestas obtenidas corroboran las aportadas en otras preguntas de los cuestionarios. Por ejemplo, consideran que cada vez más apoyarán la edición e indexación de revistas científicas como principal canal para la difusión de la investigación y el conocimiento científico, al tiempo que implementarán acuerdos con los organismos evaluadores del profesorado para un mejor reconocimiento en ese sentido de las editoriales universitarias. También asumen que deberán profesionalizar sus plantillas y equipos editoriales para ser más competitivas, pese a los avances significativos que en este ámbito han hecho en los últimos años. Asimismo, consideran que profundizarán en el open access, superando las limitaciones que ya reconocían los expertos en anteriores preguntas, principalmente para compatibilizar dicho acceso abierto con la exigida rentabilidad de sus proyectos. Otras de las respuestas obtenidas no dejan de ser algo sorprendentes. Así, los expertos señalaban como una de 
las principales fortalezas la evaluación rigurosa que hacen de los originales en el proceso editorial, mientras que ahora afirman que la generalización de los sistemas de evaluación externa y rigurosa de los originales es la medida principal que deberán llevar a cabo las editoriales universitarias españolas en los próximos 10 años. Esta manifiesta contradicción debería ser investigada en posteriores estudios que, quizá basándose en otros métodos de investigación, aportasen luz sobre los porqués de estas opiniones. Una mayor orientación hacia el marketing intensificando la renovación temática y de formatos, una mayor internacionalización tanto en la comercialización como en los contenidos y autorías, mayores esfuerzos para certificar la calidad de sus publicaciones y reforzar colecciones temáticas de calidad, consolidación de plataformas digitales de gestión y comercialización online así como una mayor inversión en tecnología para adaptarse al modelo digital y a los nuevos canales de acceso, desarrollar más acuerdos con bibliotecas universitarias y entre el conjunto de las editoriales universitarias potenciar por ejemplo el asociacionismo o las coediciones, innovar en el diseño gráfico y editorial, mejorar la distribución e incrementar los presupuestos y recursos materiales son otras importantes mejoras que las editoriales universitarias llevarán a cabo en los próximos 10 años.

\begin{tabular}{|c|c|c|c|c|}
\hline Puesto & Ítem & Mediana & Media & Desv. \\
\hline 1 & $\begin{array}{l}\text { Generalizar los sistemas de evaluación externa y riguro- } \\
\text { sa de originales }\end{array}$ & 5 & 4.30 & 0.91 \\
\hline 2 & $\begin{array}{l}\text { Profesionalizar y apoyar la edición de revistas científicas } \\
\text { para hacerlas competitivas en los rankings y bases de } \\
\text { datos }\end{array}$ & 5 & 4.26 & 1.02 \\
\hline 3 & $\begin{array}{l}\text { Profundizar en el open access, compatibilizándolo con el } \\
\text { retorno de la inversión y la proteccípon de la propiedad } \\
\text { intelectual }\end{array}$ & 5 & 4.48 & 1.10 \\
\hline 4 & Profesionalizar las plantillas y equipos editoriales & 4.5 & 4.30 & 0.77 \\
\hline 5 & $\begin{array}{l}\text { Establecer acuerdos con los organismos evaluadores } \\
\text { del profesorado para un mejor reconocimiento de las } \\
\text { publicaciones a nivel curricular }\end{array}$ & 4.5 & 4.26 & 0.90 \\
\hline 6 & $\begin{array}{l}\text { Adaptarse en mayor grado a las demandas del público, } \\
\text { potenciando su orientación hacia el marketing y la } \\
\text { impresión bajo demanda }\end{array}$ & 4 & 3.73 & 0.71 \\
\hline 7 & $\begin{array}{l}\text { Aumentar la relación con el mercado internacional, } \\
\text { principalmente latinoamericano }\end{array}$ & 4 & 3.84 & 0.72 \\
\hline 7 & Crear y reforzar colecciones temáticas de calidad & 4 & 4.30 & 0.72 \\
\hline
\end{tabular}




\begin{tabular}{|c|c|c|c|c|}
\hline 8 & $\begin{array}{l}\text { Consolidar las plataformas digitales de gestión y comer- } \\
\text { cialización on line }\end{array}$ & 4 & 4 & 0.73 \\
\hline 8 & Incrementar la internacionalización de los autores & 4 & 4.07 & 0.73 \\
\hline 9 & $\begin{array}{l}\text { Obtener y consolidar los sellos como referentes de } \\
\text { calidad }\end{array}$ & 4 & 4.23 & 0.80 \\
\hline 9 & $\begin{array}{l}\text { Optimizar el uso de metadatos y la gestión de datos } \\
\text { masivos }\end{array}$ & 4 & 4.23 & 0.80 \\
\hline 10 & $\begin{array}{l}\text { Mejorar la promoción y comunicación, aprovechando } \\
\text { las NTIC }\end{array}$ & 4 & 3.84 & 0.82 \\
\hline 11 & $\begin{array}{l}\text { Consolidar y desarrollar acuerdos con las bibliotecas } \\
\text { universitarias }\end{array}$ & 4 & 3.84 & 0.86 \\
\hline 12 & $\begin{array}{l}\text { Perseguir una mayor especialización de los consejos } \\
\text { editoriales }\end{array}$ & 4 & 3.92 & 0.87 \\
\hline 13 & Intensificar la inversión en tecnología & 4 & 3.61 & 0.88 \\
\hline 14 & $\begin{array}{l}\text { Intensificar la renovación temática y especialización } \\
\text { de las distintas editoriales, no sólo por contenidos sino } \\
\text { también por públicos o formatos }\end{array}$ & 4 & 3.65 & 0.92 \\
\hline 14 & Potenciar el asociacionismo y el papel de la marca UNE & 4 & 3.92 & 0.92 \\
\hline 15 & Innovar en el diseño gráfico y editorial & 4 & 3.57 & 0.93 \\
\hline 16 & $\begin{array}{l}\text { Mejorar el posicionamiento en los canales de distribu- } \\
\text { ción y comercialización tradicionales }\end{array}$ & 4 & 3.96 & 0.94 \\
\hline 17 & $\begin{array}{l}\text { Intensificar las coediciones y alianzas que permitan } \\
\text { mejores contenidos, costes compartidos y mayor } \\
\text { proyección }\end{array}$ & 4 & 3.96 & 0.98 \\
\hline 18 & $\begin{array}{l}\text { Desarrollar estrategias para mejorar la reputación } \\
\text { internacional de la universidad }\end{array}$ & 4 & 3.96 & 1.02 \\
\hline 19 & Buscar más activamente autores de calidad & 4 & 3.76 & 1.05 \\
\hline 19 & $\begin{array}{l}\text { Incrementar sus presupuestos y recursos materiales } \\
\text { para ser más competitivas }\end{array}$ & 4 & 3.88 & 1.05 \\
\hline 20 & $\begin{array}{l}\text { Impulsar la edición digital, innovando en formatos y } \\
\text { canales de acceso }\end{array}$ & 4 & 3.96 & 1.06 \\
\hline 21 & $\begin{array}{l}\text { Potenciar la edición en diversos idiomas para abrir el } \\
\text { mercado }\end{array}$ & 4 & 3.57 & 1.25 \\
\hline 22 & $\begin{array}{l}\text { Mejorar su cultura gerencial y la gestión de la editorial } \\
\text { como negocio }\end{array}$ & 3.5 & 3.19 & 1.27 \\
\hline 23 & $\begin{array}{l}\text { Asumir la gestión de la mayor parte de la publicación } \\
\text { digital de su universidad, desde la web institucional } \\
\text { hasta los repositorios, revistas, etc. }\end{array}$ & 3 & 3.07 & 1.3 \\
\hline 24 & Desarrollar software específicos de edición & 2 & 2.26 & 1.16 \\
\hline
\end{tabular}

Tabla 4. Medidas que adoptarán las editoriales universitarias Fuente: elaboración propia 
Las editoriales universitarias se encuentran en España en un momento crucial para garantizar su papel como referentes en la edición científico-técnica. Tradicionalmente han sido beneficiadas por la imagen de prestigio de las universidades que las impulsaban, pero en los últimos años diversos factores han abierto nuevos retos que han debilitado ese posicionamiento. Ante esta situación, creímos útil indagar, mediante un método prospectivo como es el Delphi, en la evolución prevista para el sector a partir de las opiniones de los gestores directamente implicados en el mismo.

En primer lugar, la adaptación al modelo digital se presenta como uno de los retos más importantes para los próximos 10 años. Los nuevos hábitos de consumo digital y el auge de nuevos canales de acceso al conocimiento exigen la renovación de las tradicionales editoriales universitarias. Dada la diversidad de modelos de editoriales, algunas de ellas han encajado mejor este nuevo reto y otras están aún en proceso de acomplamiento al nuevo entorno. En cualquier caso, según sus directivos el open access se presenta como un camino de futuro, si bien las dudas al respecto no son pocas por la necesidad de combinarlo con la rentabilidad o con la adaptación de las infraestructuras y las políticas editoriales que hasta el momento han venido sirviéndoles de sustento.

En segundo lugar, la calidad es otro vector fundamental que definirá el futuro de las editoriales universitarias españolas en la próxima década. Si bien los expertos participantes en nuestro estudio consideran que la cultura de la calidad y las buenas prácticas editoriales han caracterizado tradicionalmente su labor, lo cierto es que los nuevos paradigmas de calidad y los indicadores que actualmente se manejan han puesto en cuestión el modelo de sus editoriales. El hecho de que los organismos evaluadores de la actividad investigadora de los profesores universitarios primen la publicación en revistas científicas de impacto en detrimento de los libros ha limitado el papel que venían desempeñado las editoriales. Por ello, a partir de las aportaciones de los expertos en nuestra investigación, podemos concluir que el futuro pasa por dos caminos: implementar acuerdos con los organismos evaluadores basados en sistemas que permitan cuantificar la calidad de las publicaciones de estas editoriales, potenciándose por tanto los sellos y otras certificaciones de calidad, y potenciar la edición e indexación de revistas científicas, ya que en la actualidad son el canal más valorado para la difusión de la producción científica.

En tercer lugar, tanto la adaptación al modelo digital como a los nuevos paradigmas de calidad se gestionará desde algunas limitaciones que seguirán arrastrando las editoriales universitarias en el contexto de un mercado 
en donde la competencia de los grandes grupos editoriales privados es cada vez mayor. Nos referimos principalmente a la necesidad de mejorar la profesionalización de los equipos editoriales, incrementar u optimizar los recursos que permitan la competitividad en el marco de la economía digital o flexibilizar los modelos de gestión para garantizar una mayor orientación al marketing y, por tanto, a las demandas reales de los mercados. Asimismo, la mayor internacionalización, la renovación temática y de formatos o una mejor comercialización y distribución, sin duda apoyada por los medios digitales, son medidas que las editoriales universitarias adoptarán en distinto grado en los próximos 10 años para recuperar su papel en la edición científica. La experiencia acumulada en este ámbito o la especialización y diversidad de sus fondos editoriales son puntos fuertes que supondrán un buen punto de partida para este futuro próximo, en el que las editoriales seguirán teniendo una función clave tanto como servicio a la sociedad en general, contribuyendo a la transferencia de la investigación, como al mundo universitario consolidando su papel en la publicación de materiales didácticos.

\section{REFERENCIAS}

Abadal-Falgueras, Ernest, Candela Ollé-Castellà y Silvia Redondo. 2018. "Publicación de monografías en acceso abierto por editoriales universitarias españolas". Elprofesional de la información 27 (2): 300-311. doi: 10.3145/epi.2018.mar.08

Abadal-Falgueras, Ernest y Candela Ollé-Castellà. 2012. La edición universitaria en el contexto de la ciencia abierta. Barcelona: UOC.

Aldana, Lynda. 2018. Reseña de A Review of Monograph Output of American University Presses, 2009-2013, de Joseph J. Esposito y Karen Barch. doi.org/10.1080/01462679.2017.1412690

Álvarez, Alberto. 2012. Calidad en la edición universitaria. España: UNE y Universidad de Oviedo.

Bargheer, Margo y Jutta Pabst. 2016. "Being small is not a fault': Making sense of the newer generation of German language university presses”. Learned Publishing 29: 335-341.

Borcha, Marta. 2012. "La evolución de la edicion universitaria”. Delibros 269: 18-28.

Canossa-Mendes, Joao Carlos y Juan Felipe Córdoba-Restrepo, eds. 2011. Edición universitaria en América Latina. Debates, retos y experiencias. Bogotá: EULAC.

Clare, Jennifer. 2014. "University Presses in the Digital Age: How Pace University Press and Other University Presses Can Survive and Thrive Publishing”. Research Quarterly 30 (2): 195-211. doi: 10.1007/s12109-014-9362-7

Cond, Anthony. 2016. "The university press and the academic book of the future", en The academic book of the future, editado por Rebecca Lyons y Samantha Rayner, 46-55. Basingstoke, Inglaterra: Palgrave. https://link.springer.com/ book/10.1057\%2F9781137595775 
Córdoba Restrepo, Juan Felipe. 2014. "Universidad y edición. Caminos abiertos para el debate y la crítica: textos académicos en américa latina”. Boletín Cultural y Bibliográfico 48 (86): 41-51.

Cordón-García, José Antonio. 2014. "Nuevas tendencias en el libro electrónico universitario". Anuario ThinkEPI 8: 269-276.

Cordón-García, José Antonio, Julio Alonso-Arévalo, Raquel Gómez-Díaz y José Luis Alonso-Berrocal. 2014. El Ecosistema del libro electrónico universitario, $2^{a}$ ed. Salamanca: Ediciones Universidad de Salamanca y UNE.

Cordón-García, José Antonio y Raquel Gómez-Díaz. 2010. "Edición universitaria en el contexto de la edición científica: autoría, reconocimiento y valoración”. El profesional de la información 19 (1): 28-34.

Costa, Flavia y Leandro de Sagastizábal. 2016. "Las editoriales universitarias: los caminos de la profesionalización”. Anuario Centro de Estudios Económicos de la Empresa y el Desarrollo 8: 157-182.

Chartier, Roger. 2007. "La universidad y la edición. Pasado, presente y futuro", en Innovación y retos de la edición universitaria, coordinado por Magda Polo-Pujadas, 13-28. Madrid: UNE/Universidad de La Rioja.

Das, Anup Kumar. 2015. "Mapping of university presses in India: Pattern of knowledge production and dissemination". Annals of Library and Information Studies (ALIS) 62 (2): 57-67.

Deegan, Marilyn. 2017. Academic book of the future project report: A report to The AHRC \& The British Library. London: The British Library. https://academicbookfuture.files.wordpress.com/2017/06/project-report_academic-book-of-the-future_ deegan3.pdf

Delgado López-Cózar, Emilio. 2014. Las editoriales universitarias españolas: midiendo su visibilidad e impacto. IV Jornadas-Taller de formación sobre libro electrónico Unión de Editoriales Universitarias Españolas Madrid, 6 de junio de 2014. http://www.une.es/media/Ou1/Image/webjunio2014/Emilio\%20Delgado $\% 20 \mathrm{~L} \% \mathrm{C} 3 \%$ B3pez-C $\%$ C3\%B3zar.pdf

FED (Federación de Editores Europeos). 2016. European Books Publishing Statistics. https://fep-fee.eu/European-Book-Publishing-920

Fernández-Beltrán, Francisco. 2011. "La edición universitaria como referente de calidad científica y adaptación tecnológica”. Delibros 249, ejemplar dedicado a Anuario del Libro: 85-86.

Gaitán-Moya, Juan Antonio y José Luis Piñuel-Raigada. 1998. Técnicas de investigación de comunicación social. Madrid: Editorial Síntesis.

Giménez-Toledo, Elea, ed. 2018. Estudio cualitativo de las editoriales académicas. La percepción de la comunidad científica española. Madrid: Federación del Gremio de Editores de España (FGEE).

Giménez-Toledo, Elea. 2017a. "Reconocimiento académico del libro científico en español y de las editoriales universitarias iberoamericanas". Contraportada 1: 4048. http://es.calameo.com/read/0041507378156dd877862

Giménez-Toledo, Elea, ed. 2017b. La edición académica española. Indicadores y características. Madrid: Federación de Gremios de Editores de España (FGEE).

Giménez-Toledo, Elea, Carlos Miguel Tejada-Artigas y Jorge Mañana-Rodríguez. 2018. "Las editoriales universitarias iberoamericanas: una aproximación a su perfil y a sus procesos de selección de originales". Revista española de documentación cientifica 41 (2). doi.org/10.3989/redc.2018.2.1459 
Giménez-Toledo, Elea, Jorge Mañana-Rodríguez y Gunnar Sivertsen. 2017. "Scholarly book publishing: Its information sources for evaluation in the social sciences and humanities". Research Evaluation 26 (2): 91-101. doi: 10.1093/reseval/rvx007

Giménez-Toledo, Elea. 2016. "Assessment of journal \& book publishers in the humanities and social sciences in Spain”, en Research assessment in the bumanities, editado por Michael Oschner, Sven Hug y Hans Dieter-Daniel, 91-102. Cham: Springer International Publishing. doi: 10.1007/978-3-319-29016-4_8

Giménez-Toledo, Elea, Jorge Mañana-Rodríguez, Tim Engels, Peter Ingwersen, Janne Pölönen Gunnar Siverten, Frederick T. Verleysen y Alesia A. Zuccala. 2016. "Taking scholarly books into account: current developments in five european countries". Scientometrics 107 (2): 685-699. doi: 10.1007/s11192-016-1886-5

Giménez-Toledo, Elea, Jorge Mañana-Rodríguez y Carlos-Miguel Tejada-Artigas. 2015. "Scholarly Publishers Indicators: Prestige, specialization and peer review of scholarly book publishers”. El profesional de la información 24 (6): 855-860. doi: 10.3145/epi.2015.nov.18

Gómez-Hernández, José Antonio. 2007. "La edición universitaria en cuestión a los 20 años de la Unión de Editoriales Universitarias (UNE)”, en Innovación y retos de la edición universitaria, coordinado por Magda Polo-Pujadas, 31-35. Madrid: UNE/ Universidad de La Rioja.

Henny, Jean-Michel. 2015. L'édition scientifique institutionnelle en France. État des lieux, matière à réflexions, recommandations. París: Association des Éditeurs de la Recherche et de l'Enseignement Supérieur (AEDRES).

Jubb, Michael. 2017. Academic books and their future: A report to The AHRC and The British Library. London: The British Library. https://academicbookfuture.files. wordpress.com/2017/06/academic-books-and-their-futures_jubb1.pdf

Keene, Chris, Caren Milloy, Verena Weigert y Graham Stone. 2016. "The Rise of the New University Press: the current landscape and future directions". LIBER Annual Conference 29 Junio-1 de Julio, Helsinki, Finland.

Landeta-Rodríguez, Jon. 2002. El método Delphi. Una técnica de previsión del futuro. Barcelona: Ariel.

Lockett, Andrew y Lara Speicher. 2016. "New university presses in the UK: Accessing a mission”. Learned Publishing 29: 320-329. doi.org/10.1002/leap.1049

Luescher, Thierry M. y François Van Schalkwyk. 2018. "African university presses and the institutional logic of the knowledge commons". Learned Publishing 31: 288-29.

Mañana-Rodríguez, Jorge y Elea Giménez-Toledo. 2017. "Specialization and multidisciplinarity of scholarly book publishers: differences between Spanish University Presses and other scholarly publishers". Scientometrics 114: 19-30. doi:10.1007/s11192-017-2563-z

Mañana-Rodríguez, Jorge y Elena Giménez-Toledo. 2016. "Indexed University presses: overlap and geographical distribution in five book assessment databases". Trabajo presentado en la 21st International Conference on Science and Technology Indicators, Valencia, España, 14-16 de septiembre.

MECD (Ministerio de Educación, Cultura y Deporte). 2018. El sector del libro en España. Madrid: MECD. https://www.mecd.gob.es/dam/jcr:08c8edc0-2753-43069624-732c63843df0/sector-libro-2018.pdf 
Morotti, Valeria. 2015. "Social is the New Black? Últimas noticias sobre el mundo de las editoriales universitarias en Estados Unidos”. Trama E Texturas 27: 85-92. http://www.jstor.org/stable/26156228

Pérez-Lasheras, Antonio. 2007. "La edición universitaria en el siglo XXI", en Innovación y retos de la edición universitaria, coordinado por Magda Polo-Pujadas, 37-44 Madrid: UNE/Universidad de La Rioja.

Polo-Pujadas, Magda, coord. 2007. Innovación y retos de la edición universitaria. Madrid: UNE /Universidad de La Rioja.

Rayner, Samantha. 2016. "University Presses and Academic Publishing", en The Cambridge History of the Book in Britain, Volume VII: the twentieth century and beyond, editado por Andrew Nash, Claire Squires e Ian Willison, 470-483. Cambridge, Inglaterra: Cambridge University Press.

Ruediger Wischenbart Content and Consulting. 2017. BookMap.How Big Is Global Publishing? https://www.wischenbart.com/page-59

Sagastizábal, Leandro de, Jesús Anaya Rosique, José Castilho Marques y Luis Quevedo. 2016. De libros, conocimiento y otras adicciones: la edición universitaria en América Latina. Guadalajara, México: Universidad de Guadalajara.

UNE (Unión de Editoriales Universitarias Españolas). 2018. Las editoriales universitarias en cifras 2017. Madrid: UNE. http://www.une.es/media/Ou1/Image2/ webnoviembre2018/Informe_Comercio_Interior_UNE_2017.pdf

Watkinson, Charles. 2016. "Why Marriage Matters: A North American Perspective on Press/Library Partnerships”. Learned Publishing 29: 342-347. doi.org/10.1002/ leap.1044

Whitey, Lynne, Steve Cohn, Ellen Faran, Michael Jensen, Garrett Kiely, Will Underwood, Bruce Wilcox, Richard Brown, Peter Givler, Alex Holzman y Kathleen Keane. 2011. "Sustaining scholarly publishing. University presses and emerging business models. A report of the AAUP Task Force on Economic Models for Scholarly Publishing”. Journal of Scholarly Publishing 42 (4): 397441. doi: 10.3138/jsp.42.4.397

Para citar este texto:

Baladrón-Pazos, Antonio J. y Beatriz Correyero-Ruiz. 2019. "El futuro de las editoriales universitarias en España”. Investigación Bibliotecológica: archivonomía, bibliotecología e información 33 (81): 179-200. http://dx.doi.org/10.22201/iibi.24488321xe.2019.81.58057 